\section{Estresse e satisfação de pais com o atendimento em unidade de terapia intensiva neonatal}

\author{
Aila Alves Alvarenga Taurisano \\ Sonia Regina Fiorim Enumo \\ Helena Bazanelli Prebianchi \\ André Luiz Monezi Andrade
}

\section{RESUMO}

Introdução. 0 estresse dos pais devido à internação de um filho em Unidade de Terapia Intensiva Neonatal (UTIN) pode ter consequências físicas e emocionais importantes nos cuidadores. Objetivos. Analisar as relações entre o estresse dos pais e seus níveis de satisfação com o atendimento. Método. A amostra foi composta por 32 pais que tiveram seus filhos internados em UTIN. Os cuidadores preencheram um questionário sociodemográfico além dos instrumentos PSS: NICU e EMPATHIC-N. Os dados foram analisados a partir de estatística descritiva e inferencial. Resultados. Observou-se um estresse moderado a partir do instrumento PSS:NICU, especialmente nos domínios "Alteração no papel de pai/mãe" e "Número total de experiências" $(p<0,05)$. Também se detectou correlação positiva entre a idade dos pais e o nível geral de estresse $(r=0,34 ; p<0,05)$. A satisfação dos pais apresentou níveis elevados em todos os domínios. Conclusão. Os dados indicaram que quanto maior a satisfação na avaliação das equipes, menor o estresse dos pais no ambiente da UTIN.

Palavras-chave: Unidades de Terapia Intensiva; Estresse; Satisfação; Período perinatal.

\section{ABSTRACT}

\section{Stress and satisfaction of parents with the neonatal intensive care unit}

Introduction. Parents' stress due to the hospitalization of a child in a Neonatal Intensive Care Unit (NICU) can have important physical and emotional consequences on caregivers. Goals. To analyze the relationship between parents' stress and their levels of satisfaction with care. Method. The sample consisted of 32 parents who had their children hospitalized in NICUs. Caregivers completed a sociodemographic questionnaire in addition to the PSS instruments: NICU and EMPATHIC-N. Data were analyzed from descriptive and inferential statistics. Results. Moderate stress was observed from the PSS: NICU instrument, especially in the areas "Change in the role of father and mother" and "Total number of experiences" ( $p<0.05)$. A positive correlation was also found between the parents' age and the general level of stress $(r=0.34, p<0.05)$. Parents' satisfaction was high in all areas. Conclusion. The data indicated that the higher the satisfaction in the evaluation of the teams, the lower the stress of the parents in the NICU environment.

Keywords: Intensive Care Units; Stress; Satisfaction; Perinatal period.

\section{Sobre os Autores}

A. A. A. T.

https://orcid.org/0000-0001-

5457-5414

Pontifícia Universidade Católica de Campinas- Campinas, SP ailaalvarenga@gmail.com

\section{S. R. F. E.}

https://orcid.org/0000-0001-

9038-6151

Pontifícia Universidade Católica de Campinas- Campinas, SP sonia.enumo@puc-campinas. edu.br

H. B. P.

https://orcid.org/0000-00023838-8413

Pontifícia Universidade Católica de Campinas- Campinas, SP helenaprebianchi@gmail.com

\section{A. L. M. A.}

https://orcid.org/0000-0003-

0111-8935

Pontifícia Universidade Católica de Campinas- Campinas, SP andre.andrade@puc-campinas. edu.br

\section{Direitos Autorais}

Este é um artigo aberto e pode ser reproduzido livremente, distribuído, transmitido ou modificado, por qualquer pessoa desde que usado sem fins comerciais. $O$ trabalho é disponibilizado sob a licença Creative Commons CC-BY-NC 
A Unidade de Terapia Intensiva Neonatal (UTIN), por ser um ambiente permeado de barulho, equipamentos desconhecidos, rotinas rígidas, muitas pessoas se movimentando constantemente, é considerada uma fonte de estresse (Stübe, Rosa, Pretto, Cruz, Morin \& Stumm et al., 2018; Jordão, Pinto, Machado, Costa, \& Trajano et al., 2016). Em outros termos, é uma situação que excede a capacidade e recursos financeiros e físicos dos familiares para lidar com as demandas do ambiente e pessoais (Folkman, 2011). Esta condição contribui para a ocorrência de transtorno de estresse, distúrbios do sono, fadiga, ansiedade e depressão nos pais (Kegler, Neves, Silva, Jantsch, Bertoldo \& Silva et al., 2019), especialmente quando o bebê nasce prematuro (antes de 37 semanas de gestação) e com baixo peso (menos de $2,5 \mathrm{Kg}$ ) (Schappin, Wijnroks, Uniken Venema, \& Jongmans et al., 2013).

Esse contexto novo e imprevisível para os pais, e os sentimentos e reações emocionais associados, como medo, tristeza, insegurança, culpa, por exemplo (Victório et al., 2019; Magliyah \& Razzak, 2015; Ramos, Enumo, \& Paula, 2017), especialmente no caso das mães, podem afetar o vínculo mãe-bebê e o desenvolvimento do neonato (Busse, Stromgren, Thorngate \& Thomas et al., 2013), com impactos negativos posteriores no comportamento adaptativo e emocional dos bebês (Cassiano, Gaspardo, \& Linhares, 2016). Outro evento estressor para os pais e que pode prejudicar o vínculo entre eles e o bebê é a internação prolongada e as privações de contato impostas pelo ambiente (Corrêa, Andrade, de Manzo, Couto \& Duart et al., 2015).

Ter o filho internado em uma UTIN pode acarretar problemas emocionais e alterações de saúde em longo prazo nos próprios pais (Silva, Gaspardo \& Linhares, 2018). Neste sentido, dados de um estudo americano apontaram que $44 \%$ das mães que tiveram seus filhos internados em UTIN foram diagnosticadas com Transtorno Agudo de Estresse durante a internação e que esse diagnóstico está relacionado com sintomas de Estresse Pós-Traumático que ocorreram até quatro meses após o parto (Shaw et al., 2013). Além disso, o estresse dos pais de bebês que ficam em UTIN pode atrasar o ganho de competências esperadas no papel parental, como o cuidado com a criança, influenciando a interação com o bebê e seu desenvolvimento (Spinelli, Frigerio, Montali, Fasolo, Spada, \& Mangili et al., 2016).

Os principais estressores identificados pelos pais que têm seus bebês internados em UTIN são: a separação do bebê, a necessidade de informação e comunicação, o papel e a empatia da equipe de saúde (Abuidhail, Al-Motlaq, Mrayan, \& Salameh et al., 2017; Kegler et al., 2019), além do medo da morte do bebê, do sentimento de culpa e vergonha (Barr, 2015; Ramos et al., 2017). 0 momento da notícia sobre a internação e a primeira visita à UTIN foram destacados como mais estressantes por 25 mães de bebês na UTIN, gerando respostas de coping mal adaptativas, como desamparo, mas também adaptativas, como autoconfiança, busca de suporte, acomodação e busca de informações, além do coping religioso (Ramos et al., 2017). Pais e mães relataram que estar separados de seus bebês era pior quando o quadro da criança era instável, quando estavam na enfermaria sem o filho, sentindo-se desamparados e impotentes para ajudar, enquanto outras mães estavam no mesmo lugar com eles (Kegler et al., 2019; Veronez, Borghesan, Corrêa, \& Higarashi et al., 2017). Além disso, um estudo realizado em um hospital filantrópico no sul do Brasil apontou que os pais consideram altamente estressante deixar os outros filhos para acompanhar a internação do RN internado (Pieszak, Paust, Gomes, Arrué, Neves, \& Machado et al., 2017).

Nesse contexto da UTIN, variáveis relacionadas ao trabalho dos profissionais e do ambiente afetam a satisfação dos pais com os serviços de saúde oferecidos, a qual é um elemento da qualidade assistencial (Capdevila-Cogul et al., 2012), devendo ser avaliada e considerada nas intervenções com essa população que possam introduzir mudanças na rotina e organização da unidade, a fim de oferecer um atendimento de qualidade (Chertok, McCrone, Parker, \& Leslie et al., 2014). A satisfação dos pais é um conceito multidimensional, que inclui aspectos do meio ambiente e humanos. Na questão ambiental, são relevantes fatores como o mobiliário, a iluminação, o espaço físico, a organização da rotina e do acesso ao bebê. Em estudo com 87 pais, Capdevila-Cogul et al. (2012) verificaram que os pais cujos filhos estavam em um estado mais grave apreciam a acessibilidade e são críticos em relação ao conforto. Um dos preditores da satisfação dos pais foi o tempo de chegada até a UTIN e a ausência de luz e ruído, quando a internação é prolongada.

Os aspectos humanos da assistência também contribuem para a satisfação dos pais, incluindo a comunicação da equipe com os pais. Pieszak et al. (2017) observaram que pais relataram considerar humanizada a atuação que preza pelas orientações, fortalecimento do vínculo entre eles e o RN e efetivas relações interpessoais. Em outro estudo realizado por pesquisadores Sauditas, observou-se que os pais que não receberam suporte da equipe no manejo com seus filhos, apresentaram altos níveis de estresse, ansiedade e insatisfação com o serviço que lhes foi prestado (Magliyah \& Razzak, 2015)

0 provimento de informações aos pais também faz 
parte das ações de humanização (Lantz, 2017; Ottosson \& Lantz, 2017). Há dois estudos realizados na Suécia que aplicaram o instrumento EMPATHIC-N, sendo que o primeiro avaliou a satisfação dos pais apenas com relação a sua participação (Ottosson \& Lantz, 2017), e o segundo avaliou a satisfação dos pais com relação à informação (Lantz, 2017). O primeiro estudo obteve como resultado que, quanto mais prematuros os recém-nascidos (RN), maior a satisfação com a equipe de saúde e com sua participação nos cuidados com o RN (Ottosson \& Lantz, 2017). No outro trabalho, Lantz (2017) observou que pais de RNs mais prematuros apresentaram escores gerais mais elevados no domínio Informação do que pais de RNs com idade gestacional maior. Um estudo realizado com pais de RNs internados em uma UTIN do interior do Rio Grande do Sul apontou que os pais estavam satisfeitos com a assistência que receberam, demonstrando a necessidade de serem incluídos nas decisões acerca dos cuidados de seus filhos (Schmidt, Mello, Rosseto \& Souza et al., 2010). Neste mesmo trabalho, os autores também detectaram níveis elevados de satisfação com as informações recebidas pela equipe de enfermagem, indicando que esta exerce um papel fundamental no enfrentamento da internação e no aprendizado dos cuidados específicos que os pais devem ter com seus filhos.

Existem mais autores que avaliam o estresse dos pais na UTIN (Schappin et al., 2013), mas poucos analisam diretamente as relações entre estresse e satisfação dos pais nesse ambiente (CanoMoroba, Marsellés-Vidal, \& Jové-Monclús, 2012; Sánchez-Veracruz, Leal-Costa, Pastor-Rodríguez, \& Díaz-Agea et al., 2017; por exemplo). 0 estudo de CanoMoroba et al. (2012) teve um delineamento misto, visando a avaliar as emoções, sentimentos, experiências e o grau de satisfação dos 19 profissionais de saúde e pais de bebês internados na UTIN, por meio de observação não participante, diário de campo, entrevista e questionário. Os pais dessa amostra enfatizaram a importância das atitudes dos profissionais de saúde e a necessidade de receber informações de qualidade sobre o estado e evolução do seu bebê, destacando a confiança na equipe de saúde que ajuda a compreender e assimilar melhor as informações.

Considerando a inserção dos pais nesse contexto da UTIN, este estudo analisou as relações entre o estresse dos pais e seus níveis de satisfação com o atendimento. Teve-se como hipótese que pais com maiores níveis de estresse apresentariam menores níveis de satisfação com o atendimento na UTIN e vice-versa.

\section{MÉTODO}

\section{CARACTERÍSTICAS GERAIS DO ESTUDO}

Trata-se de pesquisa quantitativa, descritiva, transversal e correlacional.

\section{LOCAL}

A pesquisa foi realizada na Unidade de Terapia Intensiva Neonatal (UTIN) e no alojamento conjunto de um Hospital Universitário na cidade de Campinas-SP.

\section{PARTICIPANTES}

Participaram deste estudo 32 pais e mães (Midade= 28,5; DP $=6,4$ ) de crianças internados na UTIN. Os dados foram coletados ou na sala de espera da UTIN ou no setor e no alojamento conjunto. Em relação aos critérios de inclusão, foram considerados somente aqueles pais/ mães que estavam acompanhando a internação de seus filhos na UTIN há pelo menos um dia. Foram excluídos do estudo aqueles que preencheram de maneira incorreta ou incompleta os instrumentos.

\section{INSTRUMENTOS}

Foram aplicados individualmente três instrumentos:

1) Ficha de caracterização sociodemográfica dos participantes - elaborada pelos autores com os seguintes dados: nome, sexo, idade, data de nascimento, estado civil, escolaridade, profissão, religião, condição atual de trabaIho, data de nascimento do bebê, peso do bebê ao nascer, idade gestacional ao nascer, condições clínicas atuais (ou diagnóstico), tipo de parto, naturalidade, cidade onde mora, quantidade e parentesco ou vínculo de pessoas que residem na mesma casa, quantidade e parentesco ou vínculo de pessoas que oferecem suporte;

2) Escala de Estresse Parental: Unidade de Terapia Intensiva Neonatal/Parental Stressor Scale: Neonatal Intensive Care Unit (PSS: NICU) que avalia 26 situações específicas, comumente vivenciadas no ambiente da UTIN, a partir de três subescalas: (a) Sons e imagens; (b) Aparência e comportamento do bebê; (c) Alteração no papel de 
mãe/pai. Este instrumento é respondido em uma escala do tipo Likert, com pontuação variando de 1 a 5 ( $1=$ não estressante, e 5 = extremamente estressante). A adaptação brasileira (Souza, Dupas, \& Balieiro, 2012) apresentou um coeficiente de correlação intraclasse de 0,7 e níveis de confiabilidade variando de $a=0,8$ a $a=0,92$. Os níveis de estresse foram classificados a partir de dois grupos; Nenhum/Baixo e Moderado/Alto. Esta classificação possibilitou uma melhora na qualidade das análises pois detectou-se uma robusta diferença entre os dois grupos $(F(1,30)=67,7 ; p<0,001)$ com elevado tamanho de efeito $\left(\eta^{2}=0,69\right)$;

3) Empoderamento dos Pais na Unidade de Cuidado Intensivo Neonatal/Empowerment of Parent in the Intensive Care - Neonatology (EMPATHIC-N) - usada para avaliar a satisfação dos pais de recém-nascidos internados. A versão Brasileira (Gomez, Vidal, \& Lima, 2017) contém 57 itens, organizados em seis domínios: (a) Informação; (b) Cuidados e tratamento; (c) Participação dos pais; (d) Organização; (e) Atitude profissional; (f) Experiência global. Esta versão brasileira apresentou elevada consistência interna $(a=0,73$ até 0,93$)$.

\section{ASPECTOS ÉTICOS}

Os procedimentos deste estudo foram realizados de acordo com a legislação do Conselho Nacional de Saúde, conforme a Resolução n. 466/2012, e as diretrizes do Comitê de Ética em Pesquisa Humana da Instituição que aprovou o estudo sob o número 2.646.598 (CAAE: 86472618.9.0000.5481).

\section{PROCEDIMENTOS}

Após a aprovação do projeto no Comitê de Ética, o projeto foi apresentado e discutido com os membros da equipe tanto da UTI Neonatal quanto aqueles trabalhavam no posto de enfermagem do alojamento conjunto. Dessa forma, os locais da coleta de dados foram a sala de espera que fica dentro da UTIN e os quartos do alojamento conjunto.

Foi realizada uma aplicação-piloto, com o objetivo de testar o tempo de resposta dos questionários e a dinâmica de coleta dos dados no ambiente da UTIN. A aplicação dos instrumentos foi realizada de forma individual para garantir a compreensão dos instrumentos e o seu preenchimento correto. 0 tempo de preenchimento dos instrumentos foi de 40 minutos, em média.

\section{ANÁLISE DE DADOS}

Para o tratamento dos dados, todas as variáveis contínuas foram previamente transformadas em Z-Score e os valores acima ou abaixo de três desvios-padrão $(D P=$ 3) foram considerados outliers e suprimidos das análises específicas, para reduzir o viés na análise dos dados. Este procedimento foi adotado com base em protocolos específicos referente ao tratamento de outliers e normalização na distribuição dos dados (Andrade et al., 2020; Cruz, Scatena, Andrade, \& De Micheli et al., 2018).

As variáveis contínuas foram avaliadas pela Análise de Variância de uma via e a homogeneidade das variâncias foi avaliada pelo Teste de Levene. As demais variáveis foram analisadas a partir dos testes de Qui-quadrado (x2) ou Teste Exato de Fischer. A análise do tamanho do efeito das variáveis categóricas foi realizada pelo Teste Cramer's $V$ e das variáveis contínuas pelo Teste de Eta Squared com base nos graus de liberdade (Cunha, Enumo, Machado \& Andrade et al., 2018; Frade, De Micheli, Andrade \& Souza-Formigoni, et al., 2013). Em todas as análises, o nível de significância foi de $5 \%$.

\section{RESULTADOS}

Em relação aos dados sociodemográficos, a maioria dos participantes era mulheres, casadas ou em união estável, residentes em uma cidade de grande porte do interior paulista. A maior parte dos participantes também afirmou possuir religião, sendo que quase metade da amostra se declarou evangélica. A Tabela 1 apresenta os dados sociodemográficos a partir do nível de estresse dos participantes. Para todas as variáveis analisadas, não foram detectadas diferenças significantes quanto aos níveis de estresse. 
Tabela 1. Diferenças entre pais com e sem estresse em relação aos dados sociodemográficos e do bebê, em Unidade de Terapia Intensiva Neonatal [N = 32]

\begin{tabular}{|c|c|c|c|c|c|}
\hline PSS: NICU & $\begin{array}{c}\text { Sem estresse } \\
N(\%)\end{array}$ & $\begin{array}{l}\text { Estresse } \\
\quad N(\%)\end{array}$ & Teste & $\mathrm{p}$ & Efeito \\
\hline Idade $(M ; \pm D P)$ & $25,8( \pm 5,58)$ & $30,2( \pm 6,49)$ & $F(1,29)=3,88$ & 0,06 & 0,11 \\
\hline Gênero & & & $\chi^{2}=1,17$ & 0,27 & 0,19 \\
\hline Homem & $1(7,7)$ & $4(22,2)$ & & & \\
\hline Mulher & $12(92,3)$ & $14(77,8)$ & & & \\
\hline Estado civil & & & $X^{2}=1,97$ & 0,15 & 0,32 \\
\hline Casado & $7(53,9)$ & $14(77,8)$ & & & \\
\hline Solteiro & $6(46,1)$ & $4(22,2)$ & & & \\
\hline Escolaridade (a) & & & $\chi^{2}=0,62$ & 0,73 & 0,15 \\
\hline $1^{\circ} \mathrm{Grau}$ completo & $3(23,1)$ & $5(33,3)$ & & & \\
\hline $2^{\circ}$ Grau completo & $8(61,5)$ & $7(46,7)$ & & & \\
\hline Superior completo & $2(15,4)$ & $3(20)$ & & & \\
\hline Condição de trabalho & & & $\chi^{2}=0,27$ & 0,87 & 0,09 \\
\hline Do lar & $6(46,2)$ & $7(38,9)$ & & & \\
\hline Empregado & $5(38,5)$ & $7(38,9)$ & & & \\
\hline Desempregado & $2(15,3)$ & $4(22,2)$ & & & \\
\hline $\begin{array}{l}\text { Número de pessoas que } \\
\text { oferecem apoio }(M ; D P)\end{array}$ & $3,85( \pm 2,03)$ & $4,5( \pm 2,38)$ & $F(1,30)=0,64$ & & 0,02 \\
\hline IG do RN (M; DP) & $35,6( \pm 3,84)$ & $33,4( \pm 3,99)$ & $F(1,26)=2,21$ & & 0,14 \\
\hline Tipo de parto & & & $x^{2}=0,27$ & 0,60 & 0,09 \\
\hline Cesariana & $8(61,5)$ & $12(70,6)$ & & & \\
\hline Normal & $5(38,5)$ & $5(29,4)$ & & & \\
\hline
\end{tabular}

Nota. (a) 4 pessoas não responderam esta pergunta.

Os escores dos participantes nas duas escalas utilizadas mostram que os pais com níveis elevado de estresse apresentaram maiores pontuações nos domínios "Aparência e comportamento", "Alteração de papel" e na pontuação total do PSS:NICU, com elevado tamanho de efeito. Em relação à satisfação dos pais, não foram detectadas diferenças significativas em todos os domínios do instrumento EMPATHIC-N, segundo a presença ou ausência de estresse dos pais (Tabela 2). 
Tabela 2. Diferenças entre pais com estresse $[n=13]$ e sem estresse $[n=18]$ nos domínios de estresse e satisfação com a Unidade de Terapia Intensiva Neonatal.

\begin{tabular}{|c|c|c|c|c|c|c|c|}
\hline & \multicolumn{2}{|c|}{ Não estresse } & \multicolumn{2}{|c|}{ Estresse } & \multirow[t]{2}{*}{ Teste } & \multirow[t]{2}{*}{$P$} & \multirow[t]{2}{*}{ Efeitc } \\
\hline & $M$ & $\mathrm{DP}$ & $M$ & DP & & & \\
\hline \multicolumn{8}{|l|}{ PSS: NICU } \\
\hline Sons e imagens da UTIN & 1,70 & 0,71 & 2,17 & 0,84 & $F(1,30)=2,82$ & 0,10 & 0,08 \\
\hline $\begin{array}{l}\text { Aparência e comportamento do } \\
\text { bebê }\end{array}$ & 1,58 & 0,49 & 3,02 & 1,02 & $F(1,30)=23,2$ & ** & 0,43 \\
\hline Alteração de papeis dos pais & 2,48 & 1,23 & 4,52 & 0,65 & $F(1,30)=36,1$ & ** & 0,54 \\
\hline Total & 2,05 & 0,64 & 3,77 & 0,54 & $F(1,30)=67,7$ & $\star \star$ & 0,69 \\
\hline \multicolumn{8}{|l|}{ EMPATHIC-N } \\
\hline Informação & 5,37 & 0,73 & 5,60 & 0,48 & $F(1,30)=1,08$ & 0,30 & 0,03 \\
\hline Cuidados e tratamento & 5,66 & 0,42 & 5,77 & 0,31 & $F(1,30)=0,67$ & 0,41 & 0,02 \\
\hline Participação pais & 5,88 & 0,25 & 5,65 & 0,52 & $F(1,30)=2,24$ & 0,14 & 0,07 \\
\hline Organização & 5,51 & 0,56 & 5,76 & 0,32 & $F(1,30)=2,48$ & 0,12 & 0,07 \\
\hline Atitude profissional & 5,59 & 0,33 & 5,73 & 0,33 & $F(1,30)=0,70$ & 0,40 & 0,02 \\
\hline Experiência global & 5,39 & 1,19 & 5,77 & 0,62 & $F(1,30)=1,38$ & 0,24 & 0,04 \\
\hline Total geral & 5,57 & 0,44 & 5,71 & 0,26 & $F(1,30)=1,32$ & 0,25 & 0,04 \\
\hline Avaliação da atuação dos médicos & 8,64 & 2,34 & 9,22 & 0,94 & $F(1,30)=0,91$ & 0,34 & 0,03 \\
\hline $\begin{array}{l}\text { Avaliação da atuação dos enfer- } \\
\text { meiros }\end{array}$ & 8,57 & 2,06 & 9,16 & 0,92 & $F(1,30)=1,19$ & 0,28 & 0,04 \\
\hline Avaliação geral da equipe de saúde & 8,60 & 2,19 & 9,19 & 0,84 & $F(1,30)=1,09$ & 0,30 & 0,03 \\
\hline
\end{tabular}

Nota. ${ }^{*} \mathrm{p}<0,01$

Em relação à Tabela 3, detectou-se correlação positiva entre a idade e o domínio "sons e imagens", indicando que quanto maior a idade, maior o nível de estresse apontado pelos participantes na subescala. Além disso, também se detectou correlação positiva entre a rede de apoio e o domínio "alteração no papel de pais" de modo que quanto maior a rede de apoio, maior o nível geral de estresse referido pelos pais no que diz respeito à alteração em seu papel de pai/mãe. 
Tabela 3. Correlações entre variáveis sociodemográficas e do bebê e os dominios de estresse dos pais, em Unidade de Terapia Intensiva Neonatal [ $N$ = 32]

\begin{tabular}{lcccccc}
\hline PSS: NICU & $\begin{array}{c}\text { Aparência e com- } \\
\text { portamento }\end{array}$ & \multicolumn{2}{c}{$\begin{array}{c}\text { Alteração de papel } \\
\text { de pais }\end{array}$} & \multicolumn{2}{c}{$\begin{array}{c}\text { Número total de } \\
\text { experiências }\end{array}$} \\
\hline & $\mathrm{r}$ & $\mathrm{p}$ & $\mathrm{r}$ & $\mathrm{p}$ & $\mathrm{r}$ & $\mathrm{p}$ \\
\hline $\begin{array}{l}\text { Idade dos } \\
\text { pais }\end{array}$ & 0,34 & $*$ & 0,20 & $(0,26)$ & 0,34 & $(0,06)$ \\
$\begin{array}{l}\text { Número de } \\
\text { pessoas na } \\
\text { casa }\end{array}$ & 0,31 & 0,07 & 0,04 & 0,79 & 0,14 & 0,43 \\
$\begin{array}{l}\text { Rede de } \\
\text { apoio }\end{array}$ & 0,19 & 0,29 & 0,36 & $*$ & 0,30 & 0,08 \\
$\begin{array}{l}\text { Idade gesta- } \\
\text { cional }\end{array}$ & $-0,13$ & 0,46 & $-0,04$ & 0,80 & 0,00 & 0,99 \\
$\begin{array}{l}\text { Peso do } \\
\text { recém-nas- } \\
\text { cido }\end{array}$ & $-0,14$ & 0,43 & 0,13 & 0,46 & $-0,13$ & 0,48 \\
\hline
\end{tabular}

Nota. ${ }^{*} p<0,05$.

Quando avaliadas as mesmas variáveis a partir dos níveis de satisfação (Tabela 4), detectou-se correlação negativa entre a idade gestacional e o domínio "informação", indicando que quanto maior a idade gestacional, menor a satisfação dos pais com relação às informações que receberam por parte da equipe. Outra correlação negativa foi encontrada entre a rede de apoio e o domínio "organização", indicando que quanto maior o número de pessoas que oferecem apoio durante a internação, menor é a satisfação dos pais com relação à organização da UTIN. Além disso, a correlação negativa entre a idade com o domínio "atitude profissional" indicou que quanto maior a idade do participante, menor a sua satisfação com a atitude profissional percebida da equipe de saúde

\section{DISCUSSÃO}

Este estudo analisou os níveis de estresse e a satisfação dos pais com o atendimento em UTIN e suas possíveis correlações entre estas duas variáveis. Os principais dados indicaram que não existem respostas que considerem a experiência como "não estressante", sendo que os escores totais que variaram de "pouco estressante" a "muito estressante". Assim, todos os participantes afir- maram terem tido uma experiência estressante, porém, em diferentes níveis. Esses dados são concordantes com o estudo de Stübe et al. (2018), que também usaram o instrumento PSS: NICU. Os domínios "Sons e imagens" e "Aparência e comportamento do bebê" apresentaram os menores de estresse se comparadas à subescala que aborda a alteração no papel de pai/mãe, à semelhança do estudo de Stübe et al. (2018).

A separação entre pais e bebês imposta pelo ambiente da UTIN é amplamente discutida e considerada como uma das principais fontes de estresse. Durante a internação, eles precisam abrir mão dos cuidados de seus filhos e compartilhá-los com enfermeiros, médicos e outros profissionais da equipe, evidenciando a separação do bebê e a dificuldade para se integrar ao serviço de saúde para poder cuidar da criança e desempenhar seu papel (Abuidhail et al., 2017; Cassiano et al., 2016). Para as mães, apenas assistir à equipe oferecer cuidados ao RN pode levar a um sentimento de privação da função materna, e dificultando em muitos casos a aceitação e reconhecimento dos filhos e que estas se aceitem e se reconheçam como mães (Spinelli et al., 2016).

Diante dos dados obtidos, é possível hipotetizar que os sons e imagens da UTIN, considerando que esses es- 
Tabela 4. Correlações entre variáveis sociodemográficas e do bebê e a satisfação de pais com a Unidade de Terapia Intensiva Neonatal ( $N$ = 32]

\begin{tabular}{lccccccccccccccc}
\hline $\begin{array}{l}\text { EMPATHI- } \\
\text { C-N }\end{array}$ & Informação & $\begin{array}{c}\text { Cuidados } \\
\text { tratamento }\end{array}$ & $\begin{array}{c}\text { Participação } \\
\text { dos pais }\end{array}$ & $\begin{array}{c}\text { Organiza- } \\
\text { ção }\end{array}$ & $\begin{array}{c}\text { Atitude pro- } \\
\text { fissional }\end{array}$ & $\begin{array}{c}\text { Experiência } \\
\text { global }\end{array}$ & Total geral \\
\hline & r & $p$ & $r$ & $p$ & $r$ & $p$ & $r$ & $p$ & $r$ & $p$ & $r$ & $p$ & $r$ & $p$ \\
\hline $\begin{array}{l}\text { Idade dos } \\
\text { pais }\end{array}$ & 0,19 & 0,29 & $-0,08$ & 0,63 & $-0,02$ & 0,88 & 0,15 & 0,38 & $-0,36$ & $*$ & 0,19 & 0,27 & 0,05 & 0,75 \\
$\begin{array}{l}\text { Número } \\
\text { de pesso- } \\
\text { as } \\
\text { na casa }\end{array}$ & $-0,01$ & 0,92 & 0,06 & 0,70 & $-0,26$ & 0,14 & $-0,10$ & 0,55 & 0,19 & 0,28 & 0,03 & 0,86 & 0,06 & 0,72 \\
$\begin{array}{l}\text { Rede de } \\
\text { apoio }\end{array}$ & 0,07 & 0,68 & $-0,24$ & 0,18 & $-0,21$ & 0,22 & $-0,36$ & $*$ & $-0,17$ & 0,33 & 0,03 & 0,83 & $-0,07$ & 0,69 \\
$\begin{array}{l}\text { Idade ges- } \\
\text { tacional }\end{array}$ & $-0,42$ & $*$ & $-0,17$ & 0,35 & 0,00 & 0,98 & $-0,27$ & 0,13 & 0,06 & 0,72 & $-0,10$ & 0,57 & $-0,41$ & $*$ \\
$\begin{array}{l}\text { Peso do } \\
\text { recém- } \\
\text {-nascido }\end{array}$ & $-0,28$ & 0,12 & $-0,04$ & 0,81 & 0,08 & 0,65 & $-0,16$ & 0,36 & 0,09 & 0,62 & $-0,14$ & 0,43 & $-0,22$ & 0,21 \\
\hline
\end{tabular}

Nota. ${ }^{*} p<0,05 \mathrm{v}$

tão relacionados com as máquinas e equipamentos do setor, possam influenciar no estresse moderado que os pais apresentam na subescala que avalia a alteração no papel de pai/mãe. Os equipamentos necessários ao tratamento do RN podem dificultar a aproximação e a interação entre os pais e o RN, como, por exemplo, nos momentos em que os pais não podem pegar seus filhos devido à necessidade de permanência integral dentro de uma incubadora (Kegler et al., 2019).

Nesse sentido, a equipe de saúde desempenha um papel fundamental junto aos familiares, facilitando a adaptação dos pais ao contexto hospitalar (Chertok et al., 2016 2014; Ramos et al., 2017), corroborando o estudo de Pieszak et al. (2017) que identificou que as relações mais eficientes com a equipe de saúde são indicadores de humanização por parte dos pais. Quando a equipe favorece a aproximação entre os pais e o bebê, ela incentiva a per- manência dos cuidadores na UTIN e os instrumentaliza para oferecer aos bebês os cuidados necessários após a alta (Veronez et al., 2017; Ramos et al., 2017). Desta forma, este contato pode agregar valor nos procedimentos aprendidos pelos pais no cuidado desta criança. Dentro desta perspectiva, o estudo de Magliyah e \& Razzak (2015) mostrou a importância do suporte da equipe de saúde para diminuir o estresse e a satisfação dos pais.

A correlação entre os níveis de estresse foi encontrada quando comparadas as idades dos pais com o nível de ocorrência de estresse na subescala "Sons e imagens". Assim, quanto maior a idade dos pais, maior o nível de ocorrência de estresse na subescala "Sons e imagens". $\mathrm{Na}$ literatura, o que corrobora essas hipóteses é o fato de a prematuridade (principal diagnóstico dos RNs internados na UTIN) ser bastante associada à idade materna, uma vez que mães com mais de 35 anos têm mais chan- 
ces de um parto prematuro (Pieszak et al., 2017). Pode-se hipotetizar que pais mais velhos tenham outros filhos e que já tenham vivenciado a maternidade ou paternidade fora da UTIN. Além disso, é muito estressante deixar os outros filhos em casa para permanecer na UTIN (Pieszak et al., 2017; Veronez et al., 2017).

Em relação à satisfação, observou-se maiores escores em todas as subescalas, portanto, estão altamente satisfeitos com o atendimento que recebem nessa UTIN. 0 escore mais alto foi detectado na subescala que avalia a participação dos pais. Estes achados corroboram os resultados encontrados por Schmidt et al. (2010) em que os autores identificaram que esta participação possui um papel fundamental no enfrentamento da internação e do aprendizado dos cuidados específicos que os pais devam ter com seus filhos.

Neste estudo, os pais cujos filhos apresentavam uma gravidade de risco maior, apresentaram maiores níveis de satisfação quanto às informações que recebiam pela equipe. Esses dados corroboram os achados de outros estudos usando a mesma escala de satisfação, a EMPATHIC-N (Lantz, 2017; Ottosson \& Lantz, 2017). A satisfação também se relaciona com a idade dos participantes, pois quanto mais velhos, menor a satisfação com a atitude profissional percebida da equipe de saúde. Pode-se hipotetizar que tal fato ocorreu devido ao repertório de enfrentamento possivelmente mais complexo dos pais mais velhos, considerando que estes compreendam ou desejem compreender com mais precisão todos os estímulos presentes na UTIN. A menor satisfação com a atitude profissional corrobora essa hipótese, já que cabe à equipe informar e acolher os pais, possibilitando que eles se integrem à rotina do serviço e compreendam quais são as funções dos equipamentos presentes na UTIN (Chertok et al., 2016 2014).

Pais mais jovens podem apresentar menores níveis de estresse e maior satisfação com a atitude profissional por terem menos experiência para comparar a internação de seu RN. Também hipotetiza-se que pais com idade mais elevada já tenham vivenciado o nascimento de um filho, independentemente de este ter estado ou não em uma UTIN. Neste caso, para mães multíparas em comparação com primíparas, o nascimento de mais um filho que precisou ser internado logo após seu nascimento pode ser mais estressante. Assim, é possível que estas mães apresentem maior frequência de estratégias de enfrentamento mal adaptativas de oposição, fuga e desamparo (Ramos et al., 2017).

Os dados indicaram que os participantes com maior número de pessoas em sua rede de apoio apresentaram uma quantidade maior de experiências estressantes e maiores escores na subescala "Alteração no papel de pai/mãe". A satisfação dos pais com relação à organização da UTIN foi inversamente proporcional à quantidade de pessoas que ofereciam apoio. Neste sentido, embora a rede de apoio estivesse presente, ela possivelmente não estivesse adequada à realidade vivenciada na UTIN. Pode-se hipotetizar que esta rede influenciou negativamente a experiência dos pais em UTIN através das interferências excessivas, como sugestões e opiniões contraditórias. Além disso, os pais têm que ser mediadores das informações para a rede de apoio, que também apresenta dúvidas e questionamentos sobre o quadro clínico do RN e sua internação.

É comum que parentes e amigos queiram se envolver nos cuidados que os pais devem oferecer a um RN, e essas sugestões podem influenciar negativamente 0 enfrentamento dos cuidadores diante da internação, por serem baseadas em experiências diferentes das que eles estão vivendo naquele momento. A rede de apoio vivencia a internação apenas como espectadora, e não como participante ativa da rotina da UTIN, já que a entrada de familiares é restrita nesse ambiente. Assim, as sugestões e interferências podem estar enviesadas, e prejudicar o enfrentamento dos pais que vivenciam a internação de seu RN em uma UTIN. A rede de apoio pode também apresentar estresse e ansiedade diante da internação do $\mathrm{RN}$ e, dessa maneira, sobrecarregar os pais com perguntas excessivas ou reações emocionais exacerbadas, assim como fantasias acerca do diagnóstico, prognóstico e tempo de internação do RN, questionando, muitas vezes, a competência dos pais para lidar com o processo de cuidado de seus filhos.

Ainda que os pais da amostra estudada tenham apresentado pouco estresse relacionado aos sons e às imagens da UTIN, houve correlação significativa ao comparar a satisfação dos pais com a equipe e os níveis de ocorrência de estresse e nível de estresse geral que eles apresentaram nessa subescala. É possível concluir que, quanto maior a satisfação na avaliação da equipe médica e de enfermagem, menor o estresse dos pais com relação aos sons e imagens da UTIN.

Este trabalho apresenta limitações importantes, como o tamanho amostral, a estrutura do local da coleta de dados e à extensão dos instrumentos que foram utilizados. A maior contribuição desta pesquisa está relacionada ao avanço das pesquisas sobre pais e mães de recém-nascidos internados em UTIN e seus níveis de estresse e satisfação, podendo servir de parâmetro para novas pes- 
quisas que visem avaliar quantitativamente o estresse e a satisfação de pais nesse contexto, já que a maioria dos estudos anteriores avaliam tais conceitos de forma qualitativa. Ela também buscou facilitar e contribuir para possíveis mudanças na assistência da equipe de saúde que atua em UTIN, na medida em que identifica os principais estressores para os pais nessa condição e também o que eles consideram satisfatório no atendimento oferecido a eles durante a internação.

\section{DECLARAÇÃO DE CONFLITOS DE INTERESSE}

Os autores declaram que não há conflitos de interesses.

\section{REFERÊNCIAS}

Abuidhail, J., Al-motlaq, M., Mrayan, L., \& Salameh, T. (2017). The lived experience of Jordanian parents in a Neonatal Intensive Care Unit: A phenomenological study. The Journal of Nursing Research, 25(2). https:// doi.org/10.1097/jnr.0000000000000134

Andrade, A. L. M., Kim, D. J., Caricati, V. V., Martins, G. D. G., Kirihara, I. K., Barbugli, B. C., Enumo, S.R.F., \& De Micheli, D. (2020). Validity and reliability of the Brazilian version of the Smartphone Addiction Scale-Short Version for university students and adult population. Estudos de Psicologia (Campinas), 37. e190117. http:// dx.doi.org/10.1590/1982-0275202037e190117

Barr, P. (2015). Guilt, shame and fear of death predict neonatal intensive care unit-related parental distress. Journal of Reproductive and Infant Psychology, 33(4), 402-413. https://doi.org/10.1080/02646838.2015.10 43624

Busse, M., Stromgren, K., Thorngate, L., \& Thomas, K. A. (2013). Parents' responses to stress in the neonatal intensive care unit. Critical Care Nurse, 33(4):52-9. https://doi.org/10.4037/ccn2013715

CanoMoroba, S., Marsellés-Vidal, M. A., \& Jové-Monclús, G. (2012). Un paseo por la $5^{\text {a }}$ planta. Análisis de las vivencias y el grado de satisfacción de los padres de bebés hospitalizados en la UCIN del hospital universitario Arnaude Villanueva de Lleida. Revista de Educação Inclusiva, 5(3), 17-32. Retreived from: Dialnet Plus. https://dialnet.unirioja.es/descarga/articulo/4106309.pdf
Capdevila-Cogul, E., Sánchez-Pozóna, L., Riba-García, M., Morina-Soler, D., Ríos-Guillermo, J., Porta-Ribera, R., \& Molina-Morales, V. (2012). Valoración de la satisfacción de los padres en una unidad neonatal. Anales de Pediatría (Barcelona), 77(1),12-21. https://doi.org/10.1016/j.anpedi.2011.11.009

Cassiano, R. G. M., Gaspardo, C. M., \& Linhares, M. B. M. (2016). Prematurity, neonatal health status, and later child behavioral/ emotional problems: A systematic review. Infant Mental Health Journal, 37, 274-288. https://doi.org/10.1002/imhj.21563

Chertok, I. R., McCrone, S., Parker, D., \& Leslie, N. (2014). Review of interventions to reduce stress among mothers of infants in the NICU. Advances in Neonatal Care, 14(1), 30-37. https://doi.org/10.1097/ ANC. 0000000000000044

Corrêa, A. R., Andrade, A. C. de, Manzo, B. F., Couto, D. L., \& Duart, E. D. (2015). As práticas do Cuidado Centrado na Família na perspectiva do enfermeiro da Unidade Neonatal. Escola Anna Nery, 19(4), 629-634. https:// doi.org/10.5935/1414-8145.20150084

Cruz, F. A. D., Scatena, A., Andrade, A. L. M., \& De Micheli, D. (2018). Evaluation of Internet addiction and the quality of life of Brazilian adolescents from public and private schools. Estudos em Psicologia (Campinas), 35 (2), 193-204. https://doi.org/10.1590/198202752018000200008.

Cunha, K. S., Enumo, S. R. F., Machado, W., \& Andrade, A. L. M. (2018). Risco psicossocial familiar, coping do tratamento da obesidade infantil e controle parental da alimentação. Revista Psicologia em Pesquisa, 12(3). https://doi.org/10.24879/2018001200300492

Folkman, S. (2011). Stress, health, and coping: An overview. The Oxford handbook of stress, health, and coping, 3-11.

Frade, I. F., De Micheli, D., Andrade, A. L. M., \& de Souza-Formigoni, M. L. O. (2013). Relationship between stress symptoms and drug use among secondary students. The Spanish Journal of Psychology, 16, 1-12. https://doi.org/10.1017/sjp.2013.5

Gomez, D. B. C. A., Vidal, S. A., \& Lima, L. C. S. (2017). Brazilian adaptation and validation of the Empowerment of Parents in the Intensive Care-Neonatology (EMPATHIC-N) Questionnaire. Jornal de Pediatria, 93(2), 156164. https://doi.org/10.1016/j.jpedp.2016.08.013 
Jordão, K. R., Pinto, L. A. P., Machado, L. R., Costa, L. B. V. L., \& Trajano, E. T. L. (2016). Possíveis fatores estressantes na unidade de terapia intensiva neonatal em hospital universitário. Revista Brasileira de Terapia Intensiva, 28(3), 310-314. https://doi.org/10.5935/ 0103-507X.20160041

Kegler, J. J., Neves, E. T., Silva, A. M., Jantsch, L. B., Bertoldo, C. S., \& Silva, J. H. (2019). Estresse em pais de recém-nascidos em uma Unidade de Terapia Intensiva Neonatal. Escola Anna Nery, 23(1), e20180178. https://doi.org/10.1590/2177-9465-EAN-2018-0178

Lantz, B. (2017). Information to parents in the neonatal unit. Journal of Neonatal Nursing, 23, 180-184. https://doi.org/10.1016/j.jnn.2017.02.007

Magliyah, A. F., \& Razzak, M. I. (2015). The parents' perception of nursing support in their Neonatal Intensive Care Unit (NICU) Experience. International Journal of Advanced Computer Science and Applications, 6(2), 153-158. https://doi.org/ 10.14569/IJACSA.2015.060222

Ottosson, C., \& Lantz, B. (2017). Parental participation in neonatal care. Journal of Neonatal Nursing, 23, 112118. https://doi.org/10.1016/j.jnn.2016.11.001

Pieszak, G. M., Paust, A. M., Gomes, G. C., Arrué, A. M., Neves, E. T., \& Machado, L. M. (2017). Internação de recém-nascidos prematuros: Percepções dos pais e revelações acerca do cuidar de Enfermagem. Revista da Rede de Enfermagem do Nordeste, 18(5), 591-597. https://doi.org/10.15253/2175-6783.2017000500005

Ramos, F. P., Enumo, S. R. F., \& Paula, K. M. P. (2017). Maternal coping with baby hospitalization at a Neonatal Intensive Care Unit. Paideia, 27(67), 10-19. https://doi. org/10.1590/1982-43272767201702

Sánchez-Veracruz, M. J., Leal-Costa, C., Pastor-Rodríguez, J. D., \& Díaz-Agea, J. L. (2017). Relación entre el grado de satisfacción y el nivel de estrés identificado en padres y madres con hijos ingresados en una unidad de cuidado intensivo neonatal. Enfermería Global, 16(47), 270-80. https://doi.org/10.6018/eglobal.16.3.256061

Schappin, R., Wijnroks, L., Uniken Venema, M. M. A. T., \& Jongmans, M. J. (2013). Rethinking stress in parents of preterm infants: A meta-analysis. Plos One, 8(2), e54992. https://doi.org/10.1371/journal. pone.0054992

Schmidt, K. T., Mello, F. T., Rosseto, E. G., \& Souza, S. N. D. H. (2010). Avaliação da assistência de enfermagem em unidade neonatal na perspectiva dos pais. Cogitare Enfermagem., 15(3), 460-466. https://doi. org/10.5380/ce.v15i3.18888
Silva, N. F., Gaspardo, C. M., \& Linhares, M. B. M. (2018). Developmental care approaches for mitigating stress in preterm neonates in the neonatal intensive care unit: A systematic review. Psychology and Neuroscience, 11(2), 117-131. https://doi.org/10.1037/ pne0000119

Shaw, R. J., John, N. S., Lilo, E. A., Jo, B., Benitz, W., Stevenson, D. K., \& Horwitz, S. M. (2013). Prevention of traumatic stress in mothers with preterm infants: A randomized controlled trial. Pediatrics, 132(4). https:// doi.org/10.1542/peds.2013-1331

Souza, S. R. de, Dupas, G., \& Balieiro, M. M. F. G. (2012). Adaptação cultural e validação para a língua portuguesa da Parental Stress Scale: Neonatal Intensive Care Unit (PSS:NICU). Acta Paulista de Enfermagem, 25(2), 171-176. https://doi.org/10.1590/S010321002012000200003

Spinelli, M., Frigerio, A., Montali, L., Fasolo, M., Spada, M. S., \& Mangili, G. (2016). 'I still have difficulties feeling like a mother': The transition to motherhood of preterm infants' mothers. Psychology \& Health, 31(2), 184-204. https://doi.org/10.1080/08870446.2015.1088015

Stübe, M., Rosa, M. B. C., Pretto, C. R., Cruz, C. T., Morin, P. Y., \& Stumm, E. M. F. (2018). Níveis de estresse de pais de recém-nascidos em Unidade de Terapia Intensiva Neonatal. Rev RENE, 19, e3254-e3260. https://doi. org/10.15253/2175-6783.2018193254

Veronez, M., Borghesan, N. A. B., Corrêa, D. A. M., \& Higarashi, I. H. (2017). Vivência de mães de bebês prematuros do nascimento a alta: Notas de diários de campo. Revista Gaúcha de Enfermagem, 38(2), 1-8. https:// doi.org/10.1590/1983-1447.2017.02.60911

Victório, V. M. G., Andrade, A. L. M., da Silva, A. M. B., de Lara Machado, W., \& Enumo, S. R. F. (2019). Adolescentes com Diabetes Mellitus tipo 1: estresse, coping e adesão ao tratamento. Saúde e Pesquisa, 12(1), 6375. https://doi.org/ 10.17765/2176-9206.2019v12n 1p63-75
Data de submissão: 20/08/2019 Primeira decisão editorial: 09/01/2019 Aceite em 22/01/2020 